International Journal of Neural Systems

ÓWorld Scientific Publishing Company

\title{
EXPLORING THE BRAIN RESPONSES TO DRIVING FATIGUE \\ THROUGH SIMULTANEOUS EEG AND FNIRS MEASUREMENTS

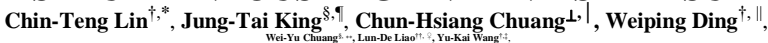 \\ ${ }^{\dagger}$ CIBCI, Centre for Artificial Intelligence,
}

FEIT, University of Technology Sydney, Sydney, 15 Broadway, Ultimo NSW 2007, Australia

$\|_{\text {dwp9988@163.com }}$

†Yukai.Wang@uts.edu.au

$\S$ Brain Research Center, National Chiao Tung University, Hsinchu 300, Taiwan

Iljtchin2@gmail.com

chchuang@mail.ntou.edu.tw

${ }^{\dagger \dagger}$ Institute of Biomedical Engineering and Nanomedicine, National Health Research Institutes, Zhunan,350, Taiwan

+1dliao@nhri.org.tw

Fatigue is one problem with driving as it can lead to difficulties with sustaining attention, behavioral lapses, and a tendency to ignore vital information or operations. In this research, we explore multimodal physiological phenomena in response to driving fatigue through simultaneous functional near-infrared spectroscopy (fNIRS) and electroencephalography (EEG) recordings with the aim of investigating the relationships between hemodynamic and electrical features and driving performance. Sixteen subjects participated in an event-related lane-deviation driving task while measuring their brain dynamics through fNIRS and EEGs. Three performance groups, classified as optimal, suboptimal, and poor, were defined for comparison. From our analysis, we find that tonic variations occur before a deviation, and phasic variations occur afterward. The tonic results show an increased concentration of oxygenated hemoglobin ( $\mathrm{HbO} 2)$ and power changes in the EEG theta, alpha, and beta bands. Both dynamics are significantly correlated with deteriorated driving performance. The phasic EEG results demonstrate event-related desynchronization associated with the onset of steering vehicle in all power bands. The concentration of phasic $\mathrm{HbO} 2$ decreased as performance worsened. Further, the negative correlations between tonic EEG delta and alpha power and $\mathrm{HbO} 2$ oscillations suggest that activations in $\mathrm{HbO} 2$ are related to mental fatigue. In summary, combined hemodynamic and electrodynamic activities can provide complete knowledge of the brain's responses as evidence of state changes during fatigue driving.

Keywords: EEG; fNIRS; fatigue driving; oxygenated hemoglobin

\section{Introduction}

Fatigue is one of the most frequent problems we confront in our daily lives. Investigating mental fatigue is especially important because it is easily induced by prolonged tasks without being noticed. ${ }^{1}$ In addition, fatigue can lead to difficulties with sustaining attention, behavioral lapses, and a tendency to ignore vital information and operations. $^{2,3}$ If people experience fatigue while driving, the consequences can vary from running off the road to drastic speed variations to fatal traffic accidents. In other words, the effects of fatigue can be much worse and more dangerous when driving than when undertaking less risky cognitive tasks. ${ }^{4-6}$

$*$ and ${ }^{\star}$ Corresponding authors. 
Recent research shows that fatigue can be accurately identified through brain dynamics. ${ }^{4-9}$ Therefore, brain activations in response to fatigue have become increasingly critical area of study over the past few decades. ${ }^{7-9}$ Further, numerous studies have explored the relationships between brain oscillations and mental fatigue through tonic or phasic EEG changes. ${ }^{5,10-14}$ Tonic variations occur before a discrete event, and phasic variations occur after the event. ${ }^{9}$ Some researchers suggest that power in the delta and theta bands increases substantially over the entire head when people drive for long periods, as does power in the alpha and beta bands but with relatively smaller augmentation. ${ }^{13}$ Others find that theta, alpha, and beta power trends in the frontal, parietal, and occipital regions are positively correlated with the level of mental fatigue. ${ }^{4,5,10-14}$ Moreover, accumulations of alpha and beta power correlate to demands on one's attention. ${ }^{11}$ Further, tonic delta, theta, and alpha activities in the parietal and occipital areas are highly correlated with deteriorating performance. ${ }^{5,12-14}$ However, EEG recordings suffer from lower spatial resolution as comparing with the other noninvasive neurophysiological devices such as functional magnetic resonance imaging (fMRI) and Magnetoencephal-ography (MEG).

Beyond EEG measurements, blood-oxygen-leveldependent imaging (BOLD-imaging) has also revealed correlations between fatigue driving and human performance. BOLD-imaging is often used as part of fMRI to measure BOLD signals while performing cognitive tasks. Previous studies have shown that fatigue causes more brain activations and greater effort is required when processing auditory information. ${ }^{15-17}$ Researchers have also reported that the higher BOLD activations could be explored from the trials with shortest reaction time (RT) in the inferior parietal lobe as performing psychomotor vigilance task. ${ }^{18,19}$ However, fMRIs restrict a person's movement to the extent that brain states during daily work or exercise cannot be explored in this way.

Over the past few decades, functional near-infrared spectroscopy (fNIRS) has emerged as an approach to reveal functional brain activity by monitoring cerebral oxygenated hemoglobin ( $\mathrm{HbO} 2)$ and deoxygenated hemoglobin (HbR). Studies drawing data from this technique have shown that subjective fatigue is negatively correlated with increased oxygenated hemoglobin over the ventrolateral prefrontal cortex when performing cognitive tasks, such as driving. ${ }^{20,21}$ Researchers have also found that $\mathrm{HbO} 2$ activation levels decline in the frontal lobe during both cognitive functions and driving performance testing after insufficient sleep. ${ }^{22}$ However, recent fatigue studies with long-duration driving tasks indicate the opposite results: $\mathrm{HbO} 2$ concentrations significantly increase in the prefrontal area when a driver feels drowsy. ${ }^{22-25}$ The contrast in results could possibly be due to the driver's effort to compensate for their weariness so as to maintain driving performance, which causes an increase in demand for $\mathrm{HbO} 2 .^{22-25 x}$ Generally, mental fatigue is negatively related to the amount of $\mathrm{HbO} 2$ in the anterior region of the brain, which can allow an increased concentration of $\mathrm{HbO} 2$ in the prefrontal cortex when participants try to maintain a desired level of performance in a task. 7,8,22,27

In this study, we designed an event-related lanedeparture driving task that mimics a realistic driving environment. Our primary aim was to explore the relationships between driver reaction times and brain dynamics. In parallel, we also wanted to examine fatigue-related brain dynamics in the occipital area, such as electrical activity and hemodynamic responses, through a multi-model integration of EEG and fNIRS signals. This allows a comprehensive investigation of the tonic and phasic brain dynamics associated with fatigue. The last purpose of the study is to gain insights into the relationships between hemoglobin changes and EEG activities.

\section{Materials and Methods}

\subsection{Participants}

In this study, 16 healthy adults (4 female), aged 22 to 26 (mean 23.5 years) with normal or corrected-to-normal vision and drivers licenses, participated in a night highway driving experiment. All participants were recruited from the National Chiao Tung University, Hsinchu, Taiwan. None reported any history of sleep or psychiatric disorders. All participants signed a consent form and were informed of the experimental procedures and the bio-signal data collection associated with the driving task in advance.

\subsection{Experimental Environment and Procedure}

One virtual reality scene was constructed with WorldToolKit (WTK) software, which is a C-based 3D 
graphic library. The driving scene was an endless fourlane road displayed on a color monitor. The program simulated driving a car at a $100 \mathrm{~km} / \mathrm{h}$ on a highway at night. The simulation was programmed to automatically drift the car away from the cruising lane with an equal probability of drifting to the left or the right. There were no other vehicles on the road or any stimuli that might influence the driver's attention. The length of the task was 60 minutes, and the entire experiment was conducted in a quiet, dimmed room to reduce distracting noise and light.

Prior to the experiment, each participant spent about five minutes reading a set of instructions and signing the consent form. They were then seated in a comfortable chair and were fitted with a suitable NIRS cap of source and detector probes. During our setup procedure, all participants were asked to practice the driving task to familiarize themselves with the experiment.

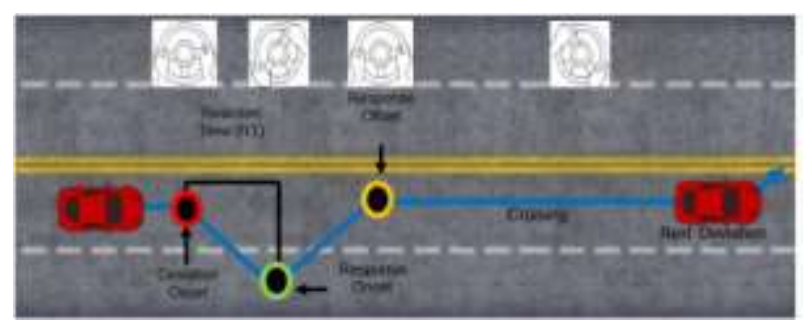

Fig. 1. The experimental protocol was 60 minutes of simulated night driving. A random deviation to the right or the left was initiated by the program every $16-20$ seconds. The participants were required to use the steering wheel to move the vehicle back to the original lane.

Once the experiment began, each participant performed the lane-keeping task for one continuous hour. The drifting events were randomly triggered, and the participants had to move the car back to the cruising lane by turning a Logitech Inc. racing wheel. The next deviation would randomly appear within 16 to 20 seconds after the vehicle had been returned to the cruising lane. The patterns of deviation were designed to fit the characteristics of hemodynamic responses. ${ }^{26}$

There are three time points in a deviation including deviation onset, response onset, and response offset, as shown in Fig. 1. Deviation onset is the moment when the program triggered the car to drift. Response onset and response offset indicate the moment the driver started and stopped turning the steering wheel to move the car back to the cruising lane after the deviation. The duration between the deviation onset and the response onset is the reaction time (RT). One epoch is defined from 2 seconds before and 15 seconds after deviation onset. The two-second time period before the stimulus was selected as the baseline (tonic), and the 15 second period after deviation onset was selected as the eventrelated change in brain dynamics (phasic).

\subsection{Data Recording}

The behavioral, EEG, and fNIRS data collected during the experiment were recorded simultaneously. RTs were continuously recorded with the WTK software, and the EEG data were recorded using a V-Amp 16 expert system (Brain Products GmbH Inc.) with 128 channels on a standard NIRS cap (https://nirx.net/nirscaps/) position according to the international 10-20 system. The EEG recordings included event markers to align the EEG data with the behavioral data. Of the 128 EEG channels, we only chose five to record EEG and fNIR signals in the occipital (POO9h and $\mathrm{POO} 10 \mathrm{~h}$ ) and parietal regions (PPO1h, Pz, and PPO2h) as represented by the purple circles in Fig. 2. This avoided duplicate fNIRS source and detector channels (Fig. 2). The EEG signals were acquired at a $2000 \mathrm{~Hz}$ sampling frequency, and the impedance of each electrode was kept below $5 \mathrm{k} \Omega$.

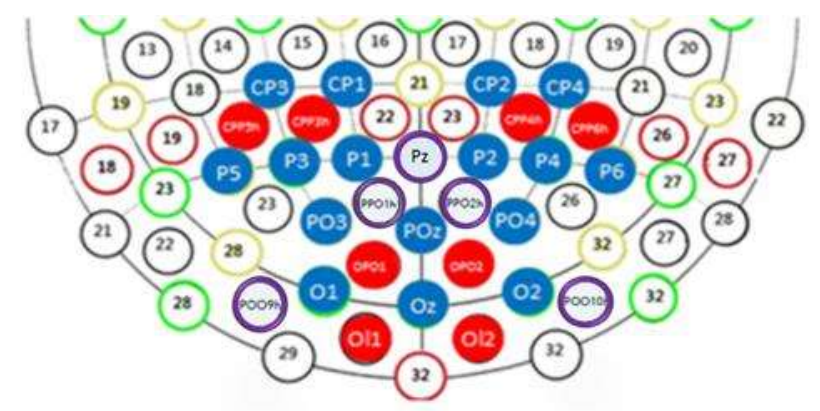

Fig. 2. EEG electrode and NIRS channel placements on a standard NIRS cap. The positions accord with the international 10-20 system. The red circles are the NIRS sources, and the blue circles are the detectors. The purple circles represent the EEG electrodes over the parietal and occipital areas.

The fNIRS data, measured concurrently, were recorded with the NIRScout apparatus (NIRx Medical Technologies, LLC.). The system consisted of 8 sources and 16 detectors to record the absorption of near-infrared light emitted by $\mathrm{HbO} 2$ and $\mathrm{HbR}$ at two wavelengths (i.e., 760 and $850 \mathrm{~nm}$ ). The sources and detectors were placed on both the left and right parietal and occipital areas using a standard NIRS cap. The exact positions are shown in 
Fig. 2. Given the area covered, we were able to use 26 channels in total: 12 channels for the occipital region, seven channels for the right parietal region, and seven channels for the left parietal region. The sampling rate of the absorption changes was $7.8125 \mathrm{~Hz}$, and the gain level of each channel was adjusted to less than or equal to 8 to ensure the quality of the measured data. Participants wore the NIRS cap that best fit their head without causing pain or discomfort. The average distance between each emitter and detector pair was approximately $3 \mathrm{~cm}$, and the depth considered to be measurable was $2-3 \mathrm{~cm}$ from the scalp.

\subsection{Data Preprocessing}

\subsubsection{EEG}

The EEG data were processed by EEGLAB ${ }^{26}$ with a band-pass filter in the range of 1 to $50 \mathrm{~Hz}$. The filtered data were then down-sampled to $250 \mathrm{~Hz}$ to reduce computational complexity. Information in the time domain was then transferred into the frequency domain by fast Fourier transform using a 128-point Welch method with 64 overlapping points. The EEG power spectral density was separated into four frequency bands (i.e., delta: 1-3 Hz; theta: 4-7 Hz; alpha: 8-12 Hz; beta: $13-30 \mathrm{~Hz})$.

\subsubsection{NIRS}

The NIRS raw data were first transformed from light absorption into relative concentration levels of $\mathrm{HbO} 2$ and HbR (unit: $\mathrm{mM}$ ) with the modified Beer-Lambert law from the nirsLAB toolbox (NIRx Medical Technologies, LLC). The $\mathrm{HbO} 2$ and $\mathrm{HbR}$ concentrations were then high-pass filtered at $0.015 \mathrm{~Hz}$ and low-pass filtered at $0.08 \mathrm{~Hz}$ to eliminate potential noise from the heartbeat, respiration, and low-frequency signal drifts. ${ }^{27-29}$. All the NIRS channels were averaged through a region analysis ${ }^{27}$ after excluding the bad channels in areas of interest to improve the signal-tonoise ratio (SNR) for further analysis.

\subsection{Data Analysis}

\subsubsection{Behavioral data analysis}

We indexed each subject's fatigue level and task performance through their RTs as per previous studies. ${ }^{10-}$ 14 If the drivers were fully concentrating, they would notice the deviation immediately and turn the car back to the cruising lane with a shorter RT, whereas, with higher levels of fatigue, their RTs would increase.

We normalized the RTs to account for the differences between subjects, rather than using the exact RT. The normalization was calculated by dividing the mean of the shortest $20 \%$ of each participant's RTs as an RT-ratio to reduce individual variance. The RT-ratios were sorted from fast to slow then used to examine the global relationships between task performance and the physiological changes in different areas of the brain.

Additionally, all trials were segmented into three groups according to performance to better investigate the relationship between the EEG data and the hemodynamic responses ${ }^{30}$. The three performance groups were:

(1) Optimal performance: RT-ratio $\leq 1$. These participants stayed alert and had the best performance.

(2) Suboptimal performance: $1<$ RT-ratio $<2$. These participants were fatigued but still maintained acceptable performance.

(3) Poor performance: RT-ratio $\geq 2$. The participants may have been too fatigued to perform the task.

\subsubsection{Tonic and Phasic analysis}

Since the characteristics of electrophysiology and hemodynamics are different, all the EEG frequency power bands and $\mathrm{HbO} 2$ concentration levels were first grouped and normalized separately into $\mathrm{z}$-scores for further comparison $(z=(x-\mu) / \sigma$, where $x$ denotes the original EEG power or $\mathrm{HbO} 2$ concentration; $\mu d e n o t e s$ the mean $(\mathrm{M})$ of the EEG power or $\mathrm{HbO} 2$ concentration; and $\mathrm{s}$ is the standard deviation of the EEG power or $\mathrm{HbO} 2$ concentration.

In the tonic analysis, the EEG and $\mathrm{HbO} 2$ concentrations within the two-second time period before deviation onset were averaged for each trial and plotted with respect to the RT-ratios.

In the phasic analysis, the mean value of the two seconds of data before deviation onset was subtracted from the event-related EEG data. Event-related spectral perturbations were used to investigate the phasic changes with behavioral performance after a vehicle deviation had occurred.

We used a moving-average technique to smooth the event-related time-frequency information. Then, the normalized EEG power and $\mathrm{HbO} 2$ concentrations for each trial were plotted with respect to the RT-ratios from 
2 seconds before deviation onset to 15 seconds after deviation onset.

All trial data were also divided into three performance groups and plotted separately.

Since NIR sources and EEG channels move when a head nods while feeling sleepy, data with a low SNR were removed. Seven subjects with high SNR EEG and NIRS signals were selected for further analysis. Moreover, due to the relatively low data scale and the potential for the data to be easily perturbed by noise, the $\mathrm{HbR}$ results are not discussed in this article. Plus, for a clear comparison with previous EEG studies ${ }^{9-14}$, only the occipital data were included for further analysis.

\subsubsection{Statistical analysis}

To statistically validate the results, we conducted Pearson's correlation tests. Each EEG frequency power and the $\mathrm{HbO} 2$ levels were tested with respect to the RTratios in the areas of interest using a bootstrap method with a threshold $p$-value of $<0.05$ for both the eventrelated spectral perturbations and the hemodynamic responses with respect to behavioral performance (i.e., the RT-ratios). A two-sample t-test was used to compare the differences in the hemodynamic responses and the power of the EEG bands among the three performance groups for both tonic and phasic analysis. All statistical analyses were completed with Matlab.

\section{Results}

\subsection{Tonic Results}

To identify the relationships between the fNIRS and EEG data as driver consciousness lapsed, we analyzed the brain dynamics in both the tonic and phasic intervals. Participants where only fNIRS data were collected were excluded from the comparisons. Fig. 3 shows the t-test correlation coefficients between the hemodynamic responses and the four EEG frequency bands in the occipital region. The results reveal significant negative correlations between the $\mathrm{HbO} 2$ concentrations and all bands (Pearson's correlation coefficient, $p<0.05$ ). For the behavioral performance, the most significant correlations were -0.57 and -0.54 with the delta and alpha bands, respectively. The theta and alpha bands also show significant correlations with the behavioral changes (see Fig. 3).

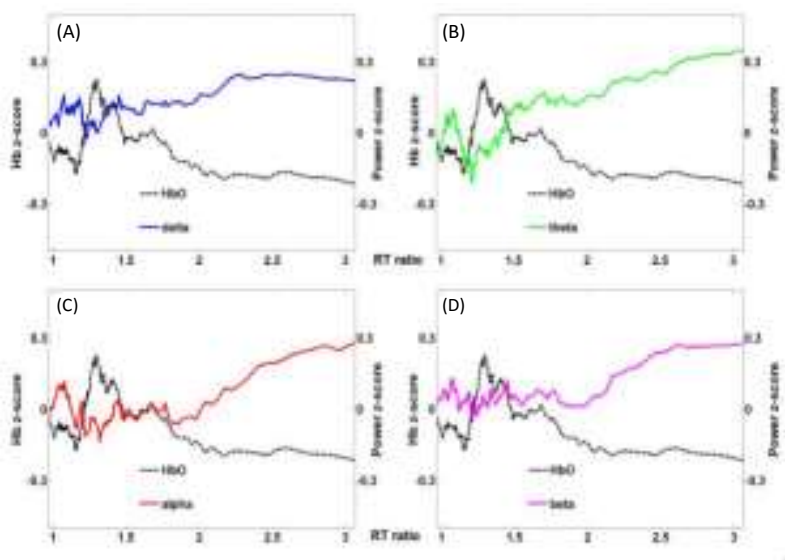

Fig. 3. The correlations between $\mathrm{HbO} 2$ concentrations and frequency changes in the occipital region. (A) delta band, (B) theta band, (C) alpha band, and (D) beta band. *: $p<0.05$

Fig. 4 shows the averaged $\mathrm{HbO} 2 \mathrm{z}$-scores in the occipital region for the three performance groups (Optimal, Suboptimal, and Poor). $\mathrm{HbO} 2$ increased in the Optimal and Suboptimal groups but decreased in the Poor group, which indicates these participants failed to maintain driving performance. There were significant differences (t-test, $p<0.05$ ) between the Optimal and Suboptimal groups and between the Suboptimal and Poor groups. The Poor performance group had the lowest $\mathrm{HbO} 2$ scores in the trials.

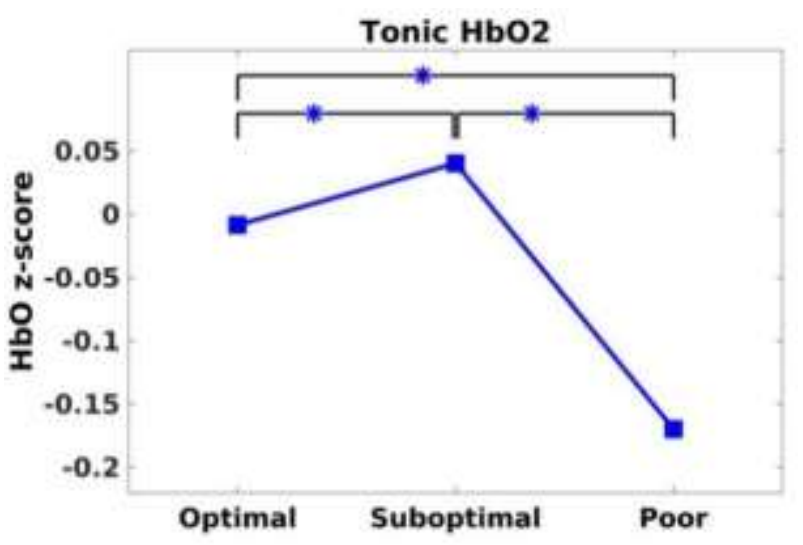

Fig. 4. Significant $\mathrm{HbO} 2$ changes in the occipital region among three performance groups (Optimal, Suboptimal, and Poor). The highest $\mathrm{HbO} 2$ scores were in the Suboptimal performance group; the lowest levels were in the Poor group. *: $p<0.05$

\subsection{Phasic Results}

The phasic power changes in the four frequency bands are shown in Fig. 5. The phasic analysis allowed us to 
observe the changes in the brain dynamics as the RTratios increased. Increases in the delta and theta bands are evident immediately after deviation onset (the black line), as are decreased perturbations before the response onset (the white curve). In the alpha and beta bands, decreased power occurred between deviation onset and response onset, and followed by increased activity.

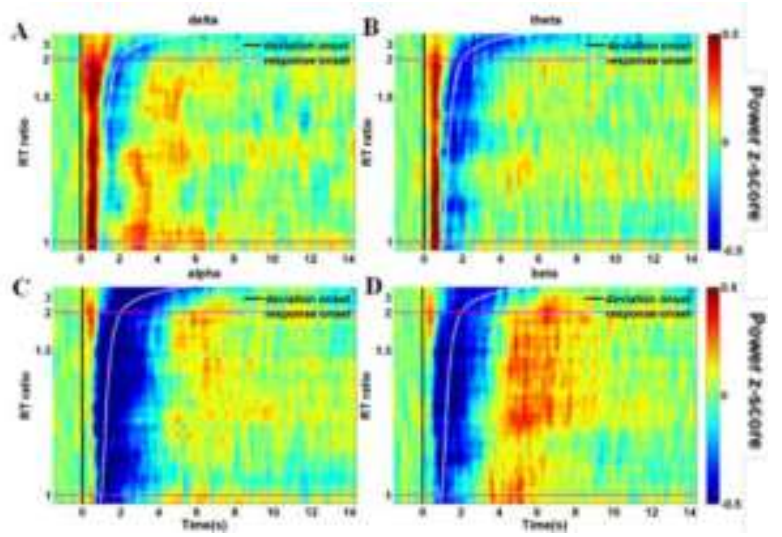

Fig. 5. The phasic changes in four frequency bands in the occipital region and the RT-ratios. The solid black lines represent the deviation inset, and the dashed white curves indicate the response onset. In each subplot, the RT-ratios are sorted (from fast to slow). The baselines were normalized. Warm (red) colors reflect increased EEG dynamics; cold (blue) colors decreased dynamics. Images are masked for significance at $p=0.05$ with FDR correction. (A) delta band (B) theta band (C) alpha band (D) beta band.

The phasic $\mathrm{HbO} 2$ responses in the occipital region with respect to the RT-ratios are illustrated in Fig. 6. Positive $\mathrm{HbO} 2$ concentration scores appeared during the 8 seconds immediately following deviance onset, after which we see negative $\mathrm{HbO} 2$ concentration scores from 8-15 seconds before the driver's $\mathrm{HbO} 2$ scores begin to return to normal (recovery stage). The positive $\mathrm{HbO} 2$ scores in the occipital region after deviance onset slightly decreased as the RT-ratio increased. Moreover, we noted that the negative $\mathrm{HbO} 2$ scores in the recovery stage tended to be lower with longer RT-ratios.

For further comparison, we analyzed the $\mathrm{HbO} 2$ concentration scores with respect to the RT-ratios across the three performance groups. After deviation onset (the black line), $\mathrm{HbO} 2$ concentrations slightly increased, followed by a decrease before returning to normal levels, as shown in Fig. 7. According to the t-test results $(p<0.05)$, the positive $\mathrm{HbO} 2$ concentration scores in the Optimal group were relatively higher than that in either the Suboptimal or Poor groups. Further, the negative $\mathrm{HbO} 2$ scores in the Suboptimal group were significantly higher than both the Poor and Optimal groups (t-test, $p<0.05$ ). Both the Suboptimal and Poor groups appeared to need more time to return back to normal $\mathrm{HbO} 2$ levels.

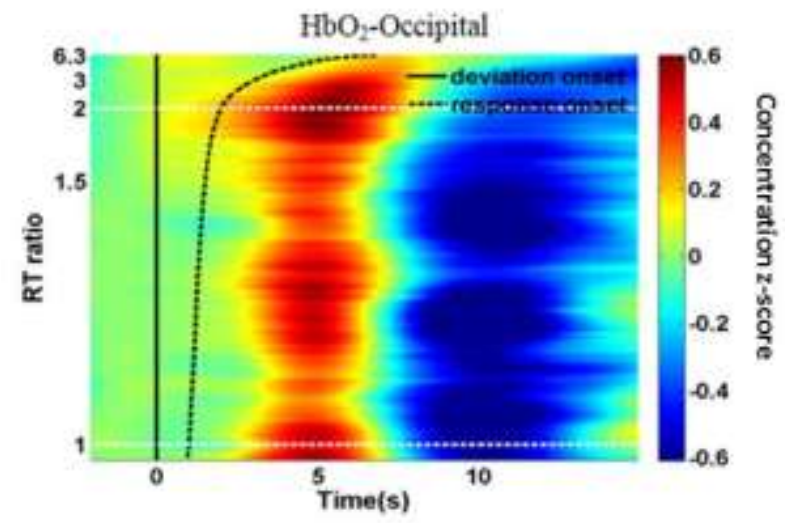

Fig. 6. Performance-related $\mathrm{HbO} 2$ concentration changes (zscores) in the occipital region. The epochs with smallest RTratios are shown at the bottom of the figure. The solid black line indicates deviation onset and the dashed white line indicates response onset. Warm colors (red) represent increased concentration levels, and cold colors (blue) indicate decreased levels. Significance at $p=0.05$ was adjusted with FDR correction.

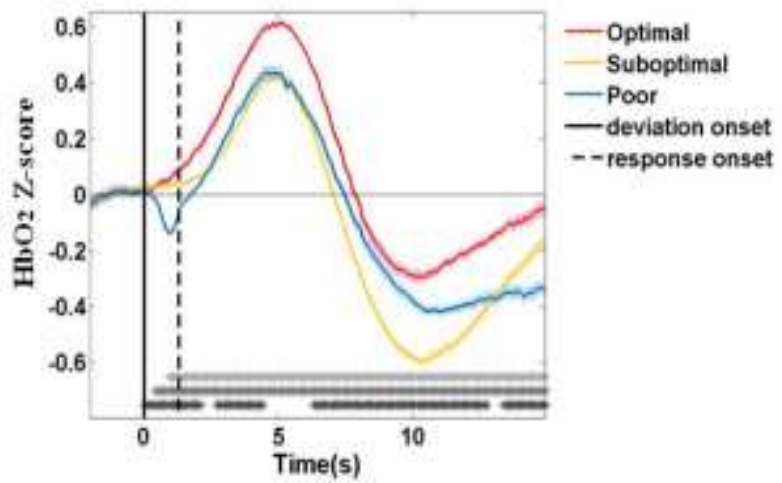

Fig. 7. The difference in occipital $\mathrm{HbO} 2$ concentration levels (zscore) after deviation onset for the three performance groups (Optimal, Suboptimal, and Poor). Significant differences $(p<0.05)$ between the groups are marked as asterisk lines at the base of the chart - light gray between the Optimal and Suboptimal groups; mid gray between the Optimal and Poor groups; black between the Suboptimal and Poor groups.

Fig. 8 presents the hemodynamic responses and the theta and alpha dynamics among the three performance groups. After deviation onset, neuronal activation appeared first, followed by changes in the $\mathrm{HbO}_{2}$ levels. 
These hemodynamic responses were related to neuronal activity in response to the events. Less theta and alpha activations were evident as the RT-ratio increased, especially in the Poor performance group. The EEG dynamics are consistent with the changes in $\mathrm{HbO}_{2}$ levels (see Figs. 6 and 7).

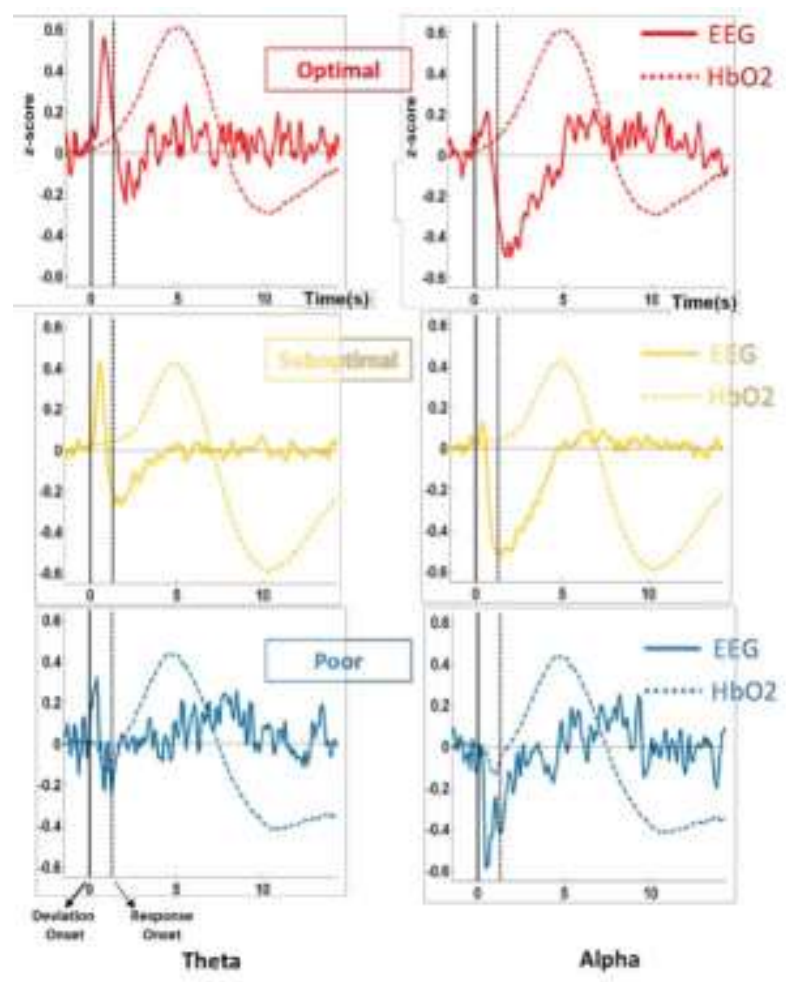

Fig. 8. Changes in EEG power and $\mathrm{HbO} 2$ concentration levels among the three performance groups (Optimal, Suboptimal, and Poor) after deviation onset (phasic comparison). Left panel: theta band. Right panel: alpha band.

\section{Discussion}

The driving experiment was designed to study brain dynamics through simultaneous fNIRS and EEG recordings in the posterior regions of the brain, particularly in the occipital area. Beyond exploring the relationships between reaction times and both biosignals, we also divided the participants into three performance groups according to optimal, suboptimal, and poor RT-ratio brackets. Comparisons between the data in each of these groups provided deeper insights into the correlations between driver fatigue and brain dynamics. A discussion on our findings and observations follows.

\subsection{Tonic Changes in EEG Signals and HbO2 Levels with Driving Performance}

According to the tonic EEG dynamics in the occipital region, power in the theta, alpha and beta bands monotonically increases as the RT-ratio increases (see Fig. 3). This positive correlation has been widely reported in previous research. ${ }^{5,11-13} \mathrm{We}$ find prolonged task-induced mental fatigue impaired driving performance and caused power deflections during the baseline period (tonic). This observation does not accord with all research, as some researchers have found a different pattern in the alpha band where alpha power initially increases and then decreases with deteriorated performance. ${ }^{10}$ The monotonic increase we observed in the alpha band could be an indication that the participants fell asleep during the experiment.

The tonic hemodynamic results (see Fig. 4) show the greatest and least changes in $\mathrm{HbO} 2$ were in the Suboptimal and Poor performance groups, respectively. The suppression of cerebral blood flow was related to subjective fatigue levels. ${ }^{16-18}$ Previous studies have also reported an increase in $\mathrm{HbO} 2$ at the onset of drowsiness. $^{7,24,31}$ The higher levels of $\mathrm{HbO} 2$ in the Suboptimal group may be associated with a low oxygen consumption rate by the brain during the transition from being alert to being drowsy. ${ }^{24,31}$ A similar phenomenon was found in the positive correlation between task difficulty and $\mathrm{HbO} 2$ levels. ${ }^{32}$ Therefore, $\mathrm{HbO} 2$ changes in the tonic domain might be illustrated by the cognitive demand of fighting fatigue. For example, in the Suboptimal group, the participants had felt tired but still tried their best to maintain driving performance, and this higher cognitive effort could be imaged. In contrast, the participants in the Poor performance group may have been so sleepy; they could only react in a passive way. As such, only a low demand would be placed on cognitive functions.

\subsection{Phasic Changes in EEG Signals and HbO2 Levels with Driving Performance}

Increased phasic theta activities and decreased phasic alpha activities associated with driving tasks have been well reported in previous EEG-related studies (see Fig. 5). ${ }^{9-13,33,46}$ The increased occipital theta activation at the beginning of the lane deviation can be interpreted as controlling cognitive demands in response to visual stimuli $^{33}$, as identifying and processing visual stimuli are associated with activations in the occipital region. ${ }^{33}$ 
Event-related desynchronization has also been discovered in the alpha and beta bands (see Fig. 5) especially, alpha suppression in the posterior area associated with visual scanning, motion preparation, and execution. ${ }^{33,34}$ In this study, the participants were asked to keep the car in the cruising lane, which meant they had to focus on the vehicle's position throughout the entire experiment as well as turn the steering wheel in response to a deviation. Among all three performance groups, the Poor performance group had the least theta activation (see Fig. 8).

We also observed positive $\mathrm{HbO} 2$ z-scores ( $\mathrm{HbO} 2$ concentration levels w.r.t. RT-ratios) in the occipital region, followed by negative scores before recovery while attempting to correct from a lane deviation (see Figs. 6 and 7). In Fig. 7, the decrease in $\mathrm{HbO} 2$ scores in the Poor performance group may be related to deteriorated driving performance. According to previous NIRS $^{19-21}$ and fMRI studies ${ }^{16-18}$, deteriorated task performance is related to reduced cerebral blood oxygen saturation.

Although the Suboptimal group had positive $\mathrm{HbO} 2$ scores post vehicle deviation (see Fig. 7), the larger negative scores of $\mathrm{HbO} 2$ can be also discovered from 8 to 15 seconds after event onset (recovery stage). The decreased $\mathrm{HbO} 2$ in the recovery stage could be due to oxygen consumption based on the reverse relation between local concentrations of deoxyhemoglobin and oxyhemoglobin. ${ }^{34-36}$ However, previous studies have suggested reduced $\mathrm{HbO} 2$ could also be associated with re-allocations in an immature cortex or high-demand on the cortex. ${ }^{37}$ Moreover, recent studies on fatigue driving with NIRS $^{8,19,22}$ and with hybrid EEG \& NIRS ${ }^{23,24}$ all demonstrate that concentrations of $\mathrm{HbO} 2$ in the prefrontal area are significantly enhanced with increased drowsiness or fatigue. Therefore, the decreased phasic $\mathrm{HbO} 2$ in the occipital site during the recovery stage could be interpreted as oxygen supplies being transferred to other task-related regions so as to meet increased demands elsewhere. In this way, all dynamics could be seen as compensatory efforts to maintain desired levels of driving performance.

In the tonic results, $\mathrm{HbO}_{2}$ oscillations were found to be inversely correlated to the delta and alpha power bands over the occipital site. Fig. 3 shows that decreased occipital alpha power was accompanied by increased cerebral oxygenation in the resting state (tonic), and vice versa. Numerous studies have attempted to reveal the physiological links between cortical activations and neuronal alpha oscillations by applying combined EEG and fMRI or fNIRS approaches. ${ }^{44,45}$ EEG alpha power has been found to be negatively correlated with hemodynamic signals over the frontal, parietal, and occipital cortical areas during the resting state. ${ }^{38}-40$ Further, spontaneous hemodynamic signal fluctuations in the visual cortex and reduced consciousness are both known to significantly increase during light sleep. This implies that when consciousness levels increase, cerebral activation will desynchronize. ${ }^{41}$ On the basis of the inverse correlations between $\mathrm{HbO} 2$ concentrations and the alpha power band, it seems possible to index the subjects' level of mental fatigue according to activations in the cerebral cortex as compared to alpha rhythms in the occipital region.

\section{Conclusions}

In this study, we explored multimodal physiological phenomena in response to fatigue driving through simultaneous EEG and fNIRS measurements while participating in a night driving experiment with random lane deviations of the vehicle. We conducted a tonic analysis of the two-second period before the lane deviation and a phasic analysis of the 15 -second period after the lane deviation. The results of the analysis reveal relationships between the EEG power spectrum and the concentration levels of $\mathrm{HbO} 2$ in the occipital region of the brain with respect to reaction times. Generally, the patterns of occipital EEG power changes in both the tonic and phasic analyses are consistent with previous findings. ${ }^{9-14}$ Spectral power increased in the theta, alpha, and beta bands as performance deteriorated, which we assume to be related to driving fatigue. ${ }^{9-14}$ Negative correlations were found between the tonic $\mathrm{HbO} 2$ concentrations and all EEG frequency bands, especially the alpha rhythms. Moreover, the phasic $\mathrm{HbO} 2$ concentrations decreased as performance worsened. The relationships between delta and alpha EEG power and the $\mathrm{HbO} 2$ oscillations suggest that activations in $\mathrm{HbO} 2$ are related to mental fatigue. Therefore, the highest tonic occipital $\mathrm{HbO} 2$ changes, which occurred in the Suboptimal group, could be because these drivers allocated more brain resources to fighting fatigue so as to maintain acceptable driving performance. Overall, we find that occipital hemodynamic and electrodynamic activities can offer complete knowledge of the brain's responses to drowsiness during driving and, therefore, 
further explorations in brain state changes associated with driver fatigue are worthwhile.

There are some limitations in the current research. First, only a few EEG and NIRS channels were measured, and only the channels in the occipital region were included in our analysis. Additional sensors covering all interesting brain regions will be considered in further research. Second, the small number of subjects may have limited our findings. Given that data quality was a major concern, the subjects with high quality of the EEG and fNIRS signals were included in this study. Third, we had discussed the prediction method in the future work to make the real contributions for fatigue driving. Despite these limitations, the preliminary results still clearly demonstrated the relationships between EEG signals, $\mathrm{HbO} 2$ levels, and driving performance.

\section{Acknowledgments}

This work was supported in part by each of: the Australian Research Council (ARC) under discovery grants DP180100670 and DP180100656; the Army Research Laboratory under Cooperative Agreement Numbers W911NF-10-2-0022 and W911NF-10-D0002/TO 0023; and the Taiwan Ministry of Science and Technology under the grant MOST 106-2221-E-009016-MY2.

\section{References}

1. K. Mandrick, Z. Chua, M. Causse, M. Causse, S. Perrey, and F. Dehais, Why a comprehensive understanding of mental workload through the measurement of neurovascular coupling is a key issue for neuroergonomics? Front Hum. Neurosci., 10 (2016) 250.

2. D. van der Linden, and P. Eling, Mental fatigue disturbs local processing more than global processing, Psychol Res., 70 (5) (2006), 395-402.

3. Y. Kato, H. Endo, and T. Kizuka, Mental fatigue and impaired response processes: event-related brain potentials in a Go/NoGo task, Int. J. Psychophysiol, 72 (2) (2009) 204-211.

4. M. A. Boksem, T. F. Meijman, and M. M. Lorist, Effects of mental fatigue on attention: An ERP study, Brain Res. Cogn., 25 (1) (2005) 107-116.

5. C. S Wei, Y. P. Lin, Y. T. Wang, C. T. Lin and T. P. Jung, A subject-transfer framework for obviating interand intra-subject variability in EEG-based drowsiness detection, NeuroImage, 174 (2018) 407-419.

6. N. Naseer1 and K. S. Hong, fNIRS-based brain- computer interfaces: A review, Front Hum. Neurosci., 9 (2015) 3.
7. M.-J. Khan, and K.-S. Hong, Passive BCI based on drowsiness detection: an fNIRS study, Biomedical Optics Express, 6(10) (2015) 4063-4078.

8. T. Liu, M. Pelowski, C. Pang, Y. Zhou, and J. Cai, Nearinfrared Spectroscopy as a Tool for Driving Research, Ergonomics, 59(3) (2016)368-79.

9. S. Makeig, and T.-P. Jung, Tonic, phasic, and transient EEG correlates of auditory awareness in drowsiness. Cognitive Brain Research, 4 (1996) 15-25.

10. K. Lal and A. Craig, Driver fatigue: electroencephalography and psychological assessment, Psychophysiology, 39 (3) (2002) 313-321.

11. C.-H. Chuang, L.-W. Ko, T.-P. Jung, and C.-T. Lin, Kinesthesia in a sustained-attention driving task, NeuroImage, 91 (2014)187-202.

12. C. T. Lin, K. C. Huang, C. F. Chao, J. A. Chen, T. W. Chiu, L. W. Ko, and T. P Jung, Tonic and phasic EEG and behavioral changes induced by arousing feedback, NeuroImage 52, (2010) 633-642.

13. K. C. Huang, T. Y. Huang, C. H. Chuang, J. T. King, Y. K. Wang, C. T. Lin, and T. P. Jung, An EEG-Based Fatigue Detection and Mitigation System, International Journal of Neural Systems, 26(4) (2016) 16500181650018.

14. A. Watanabe, N. Kato, and T. Kato, Effects of creatine on mental fatigue and cerebral hemoglobin oxygenation, Neuroscience research, 42 (4) (2002) 279-285.

15. G. Lange, J. Steffener, D. B. Cook, B. M. Bly, C. Christodoulou, W. C Liu, J. DeLuca, and B. H. Natelson, Objective evidence of cognitive complaints in Chronic Fatigue Syndrome: A BOLD fMRI study of verbal working memory, Neurolmage, 26 (2) (2005) 513-24.

16. D. B. Cook, P. J. O'Connor, G. Lange, and J. Steffener, Functional neuroimaging correlates of mental fatigue induced by cognition among chronic fatigue syndrome patients and controls, Neurolmage, 36 (1) (2007) 108-122.

17. S. P. Drummond, A. Bischoff-Grethe, and D. F. Dinges, et al., The neural basis of the psychomotor vigilance task, Sleep, 28 (9) (2005) 1059-1061.

18. J. Lim, W. C. Wu, J. Wang, J. A. Detre, D. F. Dinges, and $\mathrm{H}$. Rao, Imaging brain fatigue from sustained mental workload: An ASL perfusion study of the time-on-task effect, NeuroImage, 49 (4) (2010) 3426-3435.

19. Z. Li, M. Zhang, X. Zhang, S. Dai, X. Yu, and Y. Wang, Assessment of cerebral oxygenation during prolonged simulated driving using near infrared spectroscopy: Its implications for fatigue development, European Journal of Applied Physiology, 1073 (2009), 281-287.

20. M. Suda, M. Fukuda, T. Sato, S. Iwata, M. Song, M. Kameyama, and M. Mikuni, Subjective feeling of psychological fatigue is related to decreased reactivity in ventrolateral prefrontal cortex, Brain Research, 1252 (2009) 152-160.

21. S. Miyata, A. Noda, N. Ozaki, Y. Hara, M. Minoshima, K. Lwamoto, M. Takahashi, T. Iidaka, and Y. Koike, Insufficient sleep impairs driving performance and 
cognitive function, Neuroscience Letters, 469 (2) (2010) 229-233.

22. C. T. Lin, M. Nascimben, J. T. King and Y. K. Wang, Task-related EEG and HRV entropy factors under different real-world fatigue scenarios, Neurocomputing, 311 (2018) 24-31.

23. C. H. Chuang, Z. Cao, J. T. King, B. S. Wu, Y. K. Wang and C. T. Lin, Brain electrodynamic and hemodynamic signatures against fatigue during driving, Front Neurosci., 12 (2018) 181.

24. T. Nguyen1, S. Ahn, H. Jang, S. C. Jun, and J. G. Kim, Utilization of a combined EEG/NIRS system to predict driver drowsiness, Scientific Reports, 7 (2017) 43933.

25. L. Kocsis, P. Herman, and A. Eke, The modified BeerLambert law revisited, Physics in Medicine \& Biology, 51(5) (2006)91-8.

26. A. Delorme, and S. Makeig, EEGLAB: an open source toolbox for analysis of single trial EEG dynamics including independent component analysis, Journal of Neuroscience Methods, 134 (1) (2004) 9-21.

27. Y. K. Wang, S. A. Chen, and C. T. Lin, An EEG-based brain-computer interface for dual task driving detection, Neurocomputing, 129 (2014) 85-93.

28. S. K. Piper, A. Krueger, S. P. Koch, J. Mehnert, C. Habermehl, J. Steinbrink, H. Obrig, and C. H. Schmitz, A wearable multichannel fNIRS system for brain imaging in freely moving subjects, NeuroImage, 85 (2014) 64-71.

29. C. F. Lu, Y. C. Liu, Y. R. Yang, Y. T. Wu, and R. Y. Wang, Maintaining Gait Performance by Cortical Activation during Dual-Task Interference: A Functional Near-Infrared Spectroscopy Study, PLOS one, 10(6) (2015).

30. C.-T. Lin, C.-H. Chuang, S. Kerick, T. Mullen, T. P. Jung, L. W. Ko, S. A. Chen, J. T. King, and K. McDowell, Mind-Wandering Tends to Occur under Low Perceptual Demands during Driving, Scientific Reports, 6 (2016) 21353

31. C. Bogler, J. Mehnert, J. Steinbrink, and J. D. Haynes, Decoding vigilance with NIRS, PloS one, 9(7) (2014) e101729.

32. A. C. Merzagora, M. Izzetoglu, R. Polikar, V. Weisser, B. Onaral, and M. T. Schutheis, Functional near-infrared spectroscopy and electroenceph- alography: A multimodal imaging approach, Int. Conf. on in Foundations of augmented cognition. neuroer gonomics and operational neuroscience, San Diego, CA, USA, (2009), LNAI 5638, pp. 417-426.

33. Y. K. Wang, T. P. Jung, and C. T. Lin, Theta and alpha oscillations in attentional interaction during distracted driving, Frontiers in behavioral neuroscience, 12 (2018) 3.

34. G. Pfurtscheller, A. Stancak Jr, and C. Neuper, Eventrelated synchronization (ERS) in the alpha band-an electrophy-siological correlate of cortical idling: A review, International Journal of Psychophysiology, 24 (12) (1996) 39-46.
35. Y. Hoshi, and M. Tamura, Dynamic multichannel nearinfrared optical imaging of human brain activity. Journal of Applied Physiology, 75 (1993) 1842-1846.

36. C. Xu, B. Signe, L.-R. Allan, Functional near infrared spectroscopy (NIRS) signal improvement based on negative correlation between oxygenated and deoxygenated hemoglobin dynamics, NeuroImage, 49 (2010) 3039-3046.

37. H. Bortfeld, E. Wruck and D. A. Boas, Assessing infants' cortical response to speech using near-infrared spectroscopy, Neuroimage, 34 (2007) 407-415.

38. S.I. Goncalves, J.C. Munck, P.J.W. Pouwels, R. Schoonhoven, J.P.A. Kuijer, N.M. Maurits, J.M. Hoogduin, E.J.W. Someren, R.M. Heethaar, F.H.L. da Silva, Correlating the alpha rhythm to BOLD using simultaneous EEG/fMRI: inter-subject variability, NeuroImage, 30 (1) (2006) 203-213.

39. R. I. Goldman, J. M. Stern, J. Engel Jr, and M. S. Cohen, Simultaneous EEG and fMRI of the alpha rhythm, Neuroreport, 13 (18) (2002) 2487- 2492.

40. H. Laufs, A. Kleinschmidt, A. Beyerle, E. Eger, A. SalekHaddadi, C. Preibisch and K. Krakow, EEG- correlated fMRI of human alpha activity, NeuroImage, 19 (6) (2003) 1463-1476.

41. S. G. Horovitz, M. Fukunaga, J. A. de Zwart, P. van Gelderen, S. C. Fulton, T. J. Balkin, and J. H. Duyn, Low frequency BOLD fluctuations during resting wakefulness and light sleep: A simultaneous EEG-fMRI study, Human Brain Mapping, 29 (6) (2008) 671-682.

42. Ritter and A. Villringer, Simultaneous EEG- fMRI, Neuroscience \& Biobehavioral Reviews, 30 (6) (2006) 823-838.

43. L. Xu, B. Wang, G. Xu, W. Wang, Z. Liu, and Z. Li, Functional connectivity analysis using fNIRS in healthy subjects during prolonged simulated driving, Neuroscience Letters, 640(15) (2017) 21-28.

44. R. J. Huster, S. Debener, T. Eichele, and C. S. Herrmann, Methods for simultaneous EEG-fMRI: an introductory review. Journal of Neuroscience, 32(18) (2012) 60536060.

45. S. T. Ahn and S. C. Jung, Multi-Modal Integration of EEG-fNIRS for Brain-Computer Interfaces - Current Limitations and Future Directions, Frontiers in Human Neuroscience, 11 (2017)503.

46. Y. K. Wang, T. P. Jung and C. T. Lin, EEG-based attention tracking during distracted driving, IEEE transactions on neural systems and rehabilitation engineering, 23(6) (2015). 